\title{
A narrative review of advances in treatment and survival prognosis of HER2-positive malignant lung cancers
}

\author{
Ranpu Wu ${ }^{1}$, Bingxiao Yuan ${ }^{2}$, Chuling $\mathrm{Li}^{2}$, Zimu Wang ${ }^{3}$, Yong Song ${ }^{1,2}$, Hongbing Liu ${ }^{1,2}$ \\ ${ }^{1}$ Department of Respiratory and Critical Care Medicine, Jinling Hospital, Southeast University of Medicine, Nanjing, China; ${ }^{2}$ Department of \\ Respiratory and Critical Care Medicine, Affiliated Jinling Hospital, Medical School of Nanjing Medical University, Nanjing, China; ${ }^{3}$ Department of \\ Respiratory Medicine, Jinling Hospital, Nanjing University School of Medicine, Nanjing, China \\ Contributions: (I) Conception and design: R Wu; (II) Administrative support: None; (III) Provision of study materials or patients: None; (IV) \\ Collection and assembly of data: None; (V) Data analysis and interpretation: None; (VI) Manuscript writing: All authors; (VII) Final approval of \\ manuscript: All authors. \\ Correspondence to: Hongbing Liu, PhD; Yong Song, PhD. Department of Respiratory and Critical Care Medicine, Affiliated Jinling Hospital, Medical \\ School of Nanjing University, 305 East Zhongshan Road, Nanjing 210002, China. Email: netlhb@126.com; yong_song6310@yahoo.com.
}

\begin{abstract}
Human epidermal growth factor receptor 2 (HER2), as a receptor tyrosine kinase of EGF receptor family, whose mutation is often associated with even if less frequency but poor prognosis and shorter survival in pulmonary malignant tumor. HER2 status include mutation, overexpression, amplification and also some rare genotypes, detected by next generation sequencing (NGS), immunohistochemistry (IHC), and also fluorescence in situ hybridization (FISH). Different genotypes represent different therapeutic targets and indicate different clinical prognosis concluded by previous studies. Unfortunately, no standard guidelines for first-line treatment are widely recognized, and current therapeutic schedules include chemotherapy, radiotherapy, targeted therapy, and immunotherapy. Especially for patients with advanced metastasis, chemotherapy is based as a systemic therapy using studies of breast cancer or EGFR-positive lung adenocarcinoma as a template. Studies already explored treatment including EGFR tyrosine kinase inhibitors (TKIs) such as gefitinib and afatinib, and also trastuzumab and its conjugation like HER2-targeted antibodydrug conjugate trastuzumab emtansine (T-DM1) and conjugate trastuzumab deruxtecan (T-DXd). Also, he researches explored combination therapy with chemotherapy and TKIs or monoclonal antibodies. This review describes commonly used therapies for HER2-positive/HER2-overexpression patients and general relationship between genotypes of HER2, drug selection and final prognosis in order to provide suggestions for future diagnosis and treatment.
\end{abstract}

Keywords: Non-small cell lung cancer (NSCLC); human epidermal growth factor receptor 2 (HER2); trastuzumab; tyrosine kinase inhibitors (TKIs); chemotherapy;

Submitted Nov 09, 2020. Accepted for publication Apr 14, 2021.

doi: $10.21037 /$ jtd-20-3265

View this article at: http://dx.doi.org/10.21037/jtd-20-3265

\section{Introduction}

As one of the most lethal tumors, pulmonary malignant tumors including adenocarcinoma, squamous cell carcinoma, small cell lung cancer have always been widely concerned clinically. Human epidermal growth factor receptor 2 (HER2) receptor can overexpress or mutate in many cancers, including breast cancer, ovarian cancer, and also non-small cell lung cancer (1). HER2 overexpression and amplification have been reported in $13-20 \%$ and $2-22.8 \%$ of NSCLCs, respectively $(2,3)$. Insertion or deletion of HER2 exon 20 occurs in $2.27-4.5 \%$ of lung adenocarcinomas, especially in Asian, female patients and non-smokers (4-6). The most frequent mutation is YVMA 776-779 insertion. Insertion or deletion accounts for $90 \%$ of all HER2 gene positivity and the remaining $10 \%$ could 
be expressed as HER2 amplification (7,8). HER2 mutations are overall considered as important therapeutic targets, while trastuzumab and afatinib are already used on HER2positive patients in clinic (9). Although studies frequently find overexpression of HER2 receptor family in stage IV NSCLC patients is associated with rapid progress, brain metastasis, and also acquired resistance, whether there is a statistical difference between HER2 gene positivity and the occurrence of brain metastasis and survival of patients is under exploration (10).

However, several specific genotypes already provide reference for targeted drugs selection and prediction of its sensitivity, although different clinical trials have different criteria for patient screening classification, which has an impact on outcomes of final experiment (11). At present, HER2 amplification was found in $13 \%$ patients with acquired resistance to EGFR-mutant NSCLC, and is considered as a possible mechanism of resistance to EGFRTKIs, such as osimertinib, crizotinib (12-14). HER2 exon 20 insertion has been shown to be one of the mechanisms of resistance in EGFR-mutated advanced NSLCLC patients using osimertinib, which may explain worse clinical outcome $(15,16)$.

Even with so many attempts, the survival prognosis of HER2 mutation-positive patients remain unsatisfactory. Different status indicates different prognosis for survival and their sensitivity to different drugs is still under exploration. It can be argued that in patients with HER2 mutation, more exploration is still needed.

We present the following article in accordance with the Narrative Review reporting checklist (available at http:// dx.doi.org/10.21037/jtd-20-3265).

\section{Relationship between HER2 and prognosis}

Common genotypes of HER2 include mutation, overexpression and amplification, while common detection methods and basis for grouping patients include next generation sequencing (NGS), immunohistochemistry (IHC), and fluorescence in situ hybridization (FISH). Meanwhile, some researchers doubted that dual in situ hybridization (DISH) may be better than FISH for detection of cases with HER2 amplification (3). Some data shows that HER2 amplification and HER2 mutation usually does not overlap in patients, so they may represent different therapeutic targets (17).

HER2-positive status such as HER2 overexpression or amplification undertake worse prognostic roles. A meta- analysis of 6,135 lung cancer patients shows that HER2 overexpression determined by IHC analysis is a marker of poor prognosis in NSCLC (HR 1.48; 95\% CI: 1.22-1.80), especially in small cell lung cancer, adenocarcinoma and early NSCLC patients $(18,19)$. A multicenter study showed a median overall survival of 49.1 months in all patients with lung adenocarcinoma, while survival of HER2-negative group was just longer than that of HER2-positive group (49.3 vs. 45.0, $\mathrm{P}=0.150$ ) (20). No statistical difference was also found between HER2 mutation and patient survival in another study involving 111 patients, but HER2-negative patients tended to have longer survival $(20.5 \pm 2.4 v s$. 19.2 \pm 2.6 months, $P=0.094)(21)$, and authors speculated that this result might be due to inconsistent treatment options after recurrence or metastasis and also fewer patients number included.

In studies, immunohistochemical score is used as one of the grouping indicators to predict drug sensitivity and evaluate patient survival, with $10 \%$ adenocarcinoma scoring IHC $2+/ 3+$. Five-year survival rates of adenocarcinoma patients with IHC scores of $0,1,2$ and 3 are $75.3 \%, 77.8 \%$, $76.5 \%$ and $20.0 \%$ (22). In peter's study, grouped by IHC, patients with score 3 had a statistically lower survival rate than those with score 2 (23). No response to HER2-targeted antibody-drug conjugate trastuzumab emtansine (T-DM1) was seen in patients with score 2 , while disease control rate (DCR) was greatly reduced. This may partly explain the lack of statistical differences in data from previous clinical studies that were not grouped. However, IHC alone could not be used as a sole indicator for treatment selection, and HER2 overexpression diagnosed by IHC positivity cannot predict treatment response, calling for a scoring system to assess HER2 condition and then grouping patients (24).

\section{Specific mutations and co-mutations affect drug activity}

In a recent study, genotype G778_P780dup and G776delinsVC was found achieving greatest benefit from afatinib (25). In this study, ORR and DCR of afatinib were $15.6 \%$ and $68.8 \%$. A775_G776insYVMA group $(n=14)$ achieved an ORR of $0 \%$, DCR $35.7 \%$, and PFS was 1.2 months. While, ORR of G778_P780dup/G776delinsVC group $(n=10)$ was $40 \%$, DCR was $100 \%$, and PFS was 7.6 months. Presence of a glycine at position 778 was suggested to be a common feature of drug sensitivity mutations. Patients harboring G778_P780dup subtype achieved longer PFS (10 vs. 3.3 months, $\mathrm{P}=0.32)$ and $\mathrm{OS}$ 
(19.7 vs. 7 months, $\mathrm{P}=0.16$ ) than other 20ins but non-G778 subtypes (5).

In Yuan study (26), the clonality status of HER2 ex20ins and co-occurrence of TP53, which was the most frequently co-mutated gene in HER2 mutations (69.0\%) were identified as a potential indicator for response to afatinib, and represented shorter clinical prognosis (4). Data in Zhao's paper indicated that length of $\alpha \mathrm{C}-\beta 4$ loop and residues at HER2 776 and 778 position were also two influencing factors for sensitivity of TKIs (27). Predictably, S779_P780insVGS and G778_S779insCPG (28) were also sensitive to TKIs. Meanwhile, G776delinsAVGC with 2 amino acid extensions and retaining the G778 residue were expected to respond to dacomitinib like G778_P780dup.

Some rare mutations like transmembrane domain (TMD) mutations account for $0.13 \%$ of all lung cancer. Patients who carry HER2 TMD mutations resulted with mixed responses after receiving afatinib, asking for more effective therapeutic strategy (29). Chuang also reported that PIK3CA mutation and HER2 copy increase could be potential resistance factors to afatinib (30). Studies showed that low fragile histidine triad (FHIT) and high pHER2 phenotype could predict sensitivity to anti-HER2 therapy in NSCLC, as FHIT regulated activity of HER2 and FHITinactivated tumor cells were sensitive to HER2 inhibitors (31). Studies above conclude that selecting patients based on specific HER2 mutation and co-mutation may help improve efficacy of anti-HER2 therapy.

\section{Treatment}

At present, due to lacking guidelines for treatment of HER2-positive lung cancer patients, monoclonal antibodies for HER2-positive breast cancers like trastuzumab, EGFRTKIs and chemotherapy for lung cancers are all main drugs in clinical practice. Retrospective cohort studies comprehensively evaluate several treatment options and their clinical benefits, listed in Table 1.

\section{Trastuzumab}

\section{Trastuzumab and its conjugation}

Trastuzumab emtansine (T-DM1), a HER2-targeted antibody-drug conjugate can significantly improve survival rate of HER2 positive breast cancer, which may do the same to NSCLC patients (47). In a retrospective study involving 101 patients with HER2 exon-20 insertion from 38 centers, 5 of them with concomitant EGFR mutations, and 58 of them finally received trastuzumab or T-DM1. Median PFS in trastuzumab group was 4.8 months, and OS was 13.3 months (46). Li also assessed activity of T-DM1 on 18 patients with advanced HER2-mutant lung adenocarcinomas, with a response rate of $44 \%$ (95\% CI: 22-69\%) in patients with HER2 exon 20 insertions and a median PFS of 5 months (95\% CI: 3-9 months) (48). However, current studies have shown that only cells with strong HER2 immunostaining could be killed after exposure to Trastuzumab Emtansine (T-DM1), while cells with weak staining may escape and continue to grow. Therefore, patients with IHC 3 + could choose T-DM1 treatment, while patients with IHC $2+$ or less may not achieve better response concluded by peters' study (23). Authors analyzed that some studies on T-DM1 do not exclude PIK3CA mutation, which may also make clinical results not positive in NSCLC patients.

In Tsurutani's study (NCT02564900), HER2-targeted antibody-drug conjugate Trastuzumab deruxtecan was assessed in 60 patients with pretreated solid tumors, covering HER2-expressing and HER2-mutant NSCLC, salivary gland cancer, biliary tract cancer, breast cancer and so on. In NSCLC group, ORR was $72.7 \%(8 / 11)$, and median PFS was 11.3 months (95\% CI: 8.1-14.3\%) (49). In DestinyLung01 study covering with 42 relapsed/refractory HER2-mutant NSCLC, T-DXd led to a confirmed objective response rate (ORR) of $61.9 \%$ and median PFS was 14.0 months (50). FDA already granted a breakthrough therapy designation to T-DXd for the treatment of patients with metastatic HER2-mutant NSCLC (51).

\section{Combination of trastuzumab and others therapy}

In 2004, Lara, Zinner, and Gatzemeier used combination of trastuzumab and chemotherapy, including docetaxel, cisplatin and gemcitabine in HER2-positive lung cancer patients (32-34). Except Lara, other two studies were found clinical effectively, with a response rates (ORR) of $38 \%$, $36 \%$ and DCR of $81 \%, 80 \%$. In Gatzemeier's study, PFS was 6.1 months (95\% CI: 0.9-19.6 months) and OS was 12.2 months (95\% CI: 0.1-19.6 months).

Neratinib and pertuzumab were also found effective in HER2-positive tumor models (52,53). Follow-up studies are still needed to validate clinical benefits of trastuzumab in combination with TKIs or pertuzumab.

\section{Tyrosine kinase inbibitors (TKIs)}

In non-small cell lung cancer, TKIs are widely used in 


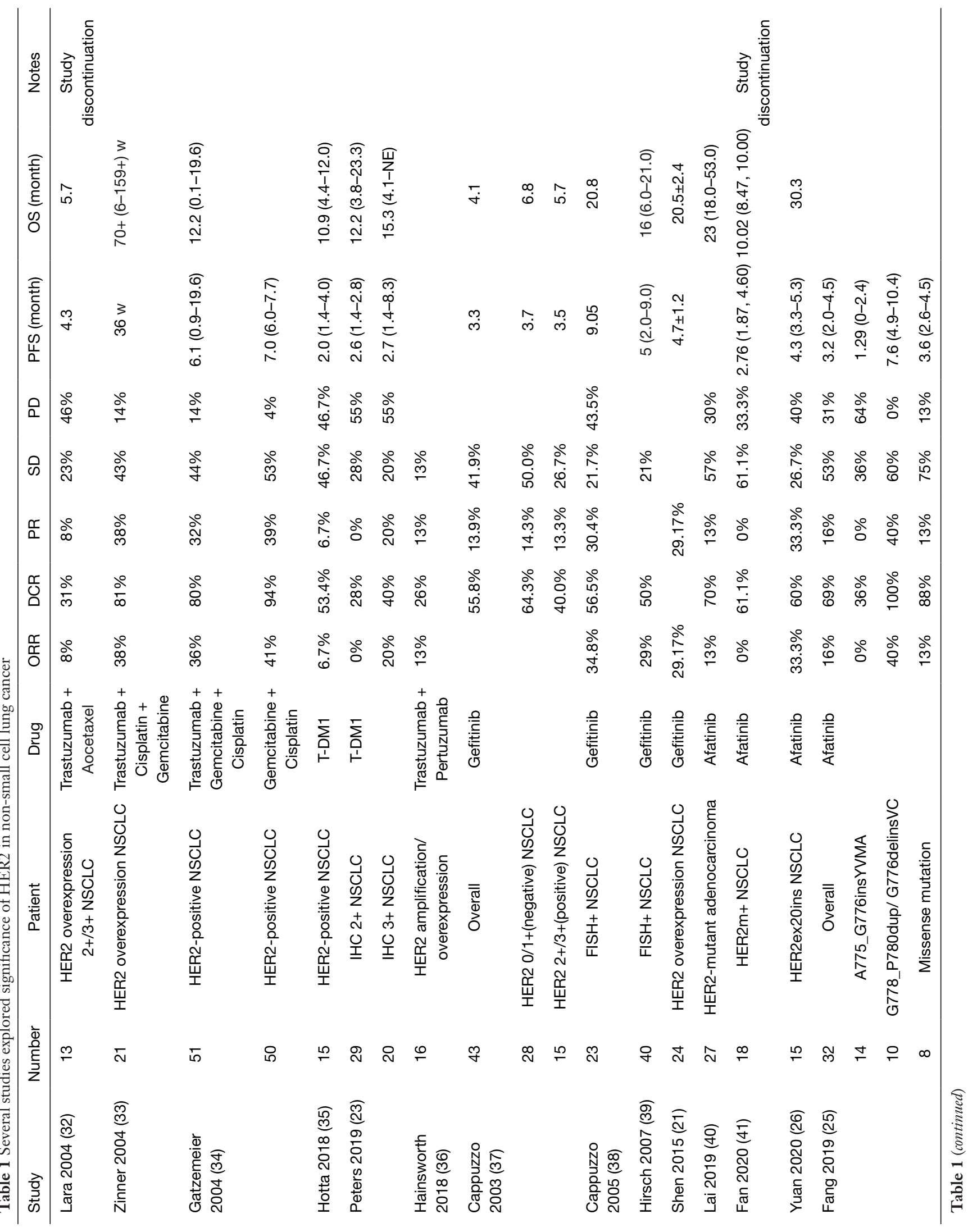




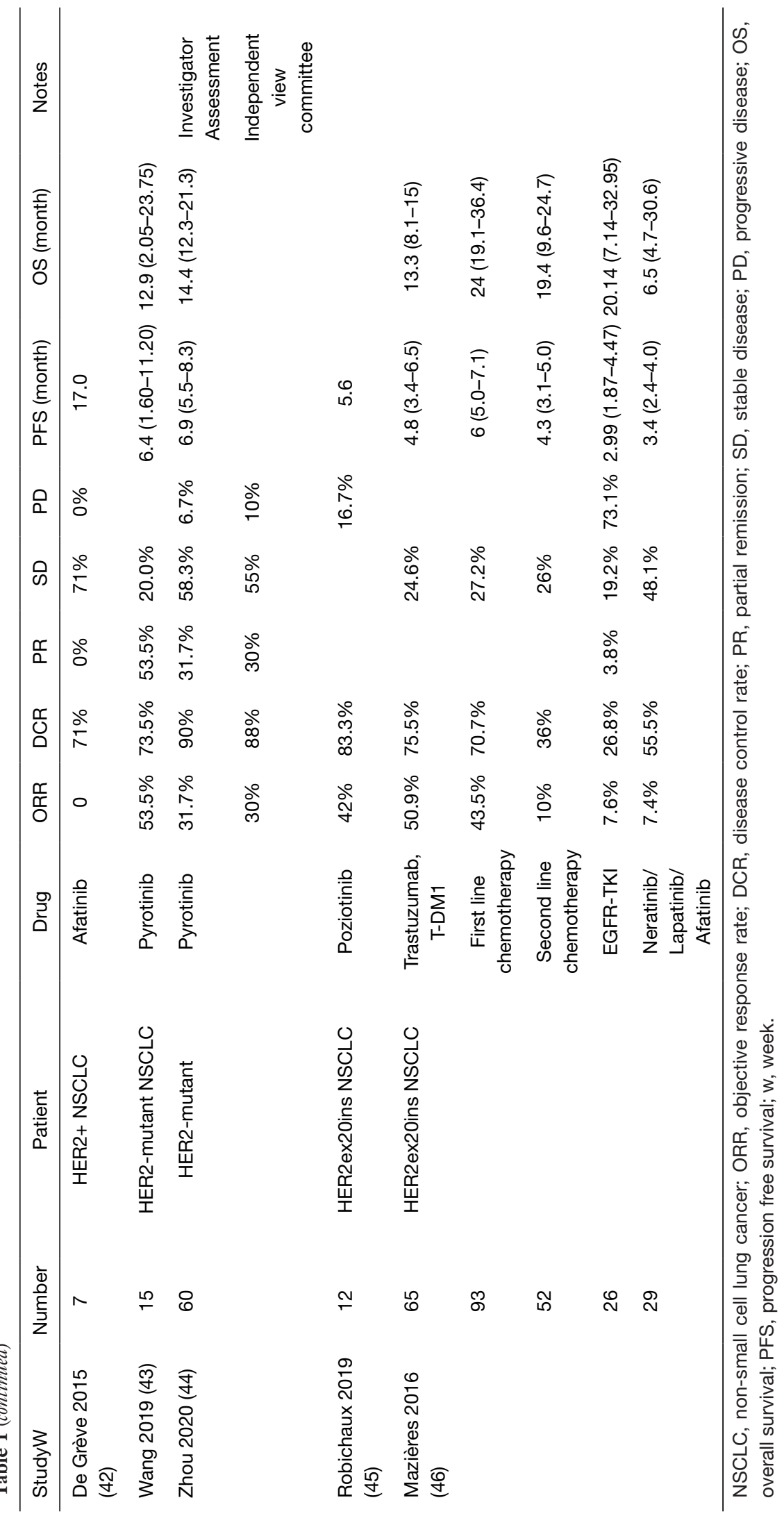


EGFR-positive patients, but the benefits of EGFR targeted drugs are far less in HER2-positive NSCLC than in EGFRpositive ones. Different genotypes of HER2 mutation shows different clinical effects towards different TKIs, such as gefitinib, afatinib and dacomitinib. Many studies are also exploring new drugs for HER2-mutated patients with or without EGFR mutation.

\section{Gefitinib}

Gefitinib, as a classic first-generation EGFR-TKI, is used as irreplaceable treatment for Lung adenocarcinoma patients with EGFR mutation like exon 19 deletion, and exon 21 (51). In 2009, Varella found HER2 FISH+ patients could benefit from gefitinib therapy. A group of 44 Japanese patients with recurrent NSCLC who received $250 \mathrm{mg}$ gefitinib daily detected 23 HER2 FISH+ patients (53\%), with an overall response rate of 52\% (54). In 106 Chinese NSCLC patients treated with gefitinib, ORR could be significantly higher in HER2-positive patients regardless of EGFR mutation status (55). However, in a retrospective study, the EGFR/HER2 status of 111 lung cancer patients were diagnosed by undergoing biopsy or surgery, combination of EGFR mutation and HER2 overexpression was $5.41 \%(n=6)$. There was no significant difference in disease control rate (DCR) and OS (5.7 vs. 6.8 months) between HER2 overexpression and HER2 negative group, with or without EGFR mutation (21). Another study involving 63 patients with EGFR mutation obtained a similar conclusion that HER2 expression levels were not associated with survival prognosis. The PFS between two groups was 4.7 and 3.9 months, $\mathrm{P}=0.085$, and $\mathrm{OS}$ was 20.5 and 19.2 months, $\mathrm{P}=0.094$ (37).

These studies demonstrated that gefitinib was not beneficial for disease control in HER2-positive patients, and some patients with HER2 mutation but without EGFR mutation may need to avoid choosing gefitinib as first-line treatment. High HER2 copy number, to some extent, may affect gefitinib's clinic effect (56), but HER2 overexpression was not useful in predicting sensitivity of NSCLC patients with gefitinib.

\section{Afatinib}

In advanced lung adenocarcinoma patients carrying HER2 mutations, studies tend to explore afatinib as first-line treatment (57). Clinical studies on afatinib have achieved preclinical model inhibition, and shown a good response rate in clinical studies (58). Lai assessed 23 patients with stage IV HER2 mutant lung adenocarcinoma, 57\% of whom achieved stable disease after receiving afatinib. Median overall survival of them was 23 months (95\% CI: 18-53 months) (40). In another study of 28 pretreated stage IV NSCLC patients, median PFS in HER2 mutant subgroup ( $\mathrm{n}=12$ ) was 9.6 months, with an ORR of $33 \%$ and a DCR of $100 \%$ (59). In Fan's study (41), 18 III b/IV NSCLC patients with HER2-mutation positive received afatinib after failing one or two prior lines of chemotherapy. 11 patients $(61.1 \%)$ had stable disease, while 6 patients (33.3\%) had progressive disease, and DCR was $61.1 \%$. Median PFS was 2.76 months (95\% CI: $1.87-4.60$ months), and OS was 10.02 months (95\% CI: 8.47-10.08 months).

Studies have shown that the median PFS of patients treated with afatinib and paclitaxel is 6.7 weeks, and afatinib plus paclitaxel may be more effective than afatinib alone (60). Combination of afatinib and chemotherapy could be a better choice for HER2 positive NSCLC patients, and survival time of HER2-TKI plus chemotherapy could not be affected by order of these two drugs $(\mathrm{P}=0.263)$. Afatinib was effective in NSCLC patients who were resistant to erlotinib or gefitinib, with a response rate of $11.6 \%$, a PFS of 3.9 months, and an overall survival of 7.3 months in 86 evaluable patients (61). Also, its clinical activity can still be seen in HER2-positive patients after several lines of pretreatment $(42,62)$. It could induce partial response in patients who have previously received other HER2 targeted therapies, including trastuzumab and pertuzumab (40).

The researches above illustrated that afatinib may be used as a choice for advanced HER2-positive patients after failure of multiline therapies (63). At the same time, afatinib also showed high efficacy in untreated patients with HER2 mutation and then could be considered for those who are not suitable for standard treatment (64).

\section{Other HER2 inhibitors in exploration}

Clinical trials are now exploring effect of some novel HER2 inhibitors like pyrotinib, poziotinib, lapatinib, dacomitinib, neratinib to prolong survival for patients out of choice after failure of monoclonal antibody or afatinib (65).

In previous studies, pyrotinib showed promising antitumor activity and acceptable safety in NSCLC patients with HER2 mutation. A patient diagnosed with lung adenocarcinoma and bone marrow metastasis achieved stable disease and recovery of pancytopenia after two months of pyrotinib therapy (66). A rare subtype G778 S779insCPG was also reported partial response to pyrotinib and PFS was 12.8 months (28). Recently, Wang's study (43) included 15 HER2 patients with an ORR of $53.3 \%$, a 
median OS of 12.9 months and most patients tolerated pyrotinib well. Zhou's study included 60 NSCLC HER2 mutated patients treated with pyrotinib monotherapy, and only one patient was confirmed combining with EGFR mutation. Median overall survival was 14.4 months $(95 \%$ CI: 12.3-21.3 months), median duration of response was 6.9 months (95\% CI: 4.9-11.1 months), and median PFS was 6.9 months (95\% CI: 5.5-8.3 months) (44).

In previous studies, poziotinib was once compared with nine TKIs in a preclinical study and was found showing most activity against HER2 exon 20 insertion mutation (67). A phase II clinical testing in HER2 exon 20-mutant NSCLC patients resulted in an ORR of $42 \%$ in the first 12 evaluable patients, and also proved poziotinib may upregulating HER2 cell-surface expression activity of T-DM1 in preclinical models (45). Authors considered that this high activity might be due to small size of poziotinib and its flexible structure enabling it to bind to kinase domain. However, poziotinib induced significant toxicity, including more than $50 \%$ of patients with grade 3 or higher toxicity and even death due to pneumonia (68).

Combination of lapatinib and trastuzumab proved to be an attemptable method for HER2-mutuated NSCLC patients (69). A total of 18 patients treated with lapatinib, partial response was detected in 4 patients, and resulted that even if lapatinib and pemetrexed were well tolerated, while clinical outcome was still similar to pemetrexed monotherapy (70). Another study of 60 NSCLC patients demonstrated that lapatinib combined with whole brain radiotherapy (WBRT) is a feasible method in patients with brain metastasis. In NSCLC group, OS was 4.2 months (95\% CI: 3.2-5.1 months) with a median TTP of 2.8 months (95\% CI: 1.7-4.1 months) (71). NSCLC patients with HER2 amplification may benefit from lapatinib alone, while this could not be determined by current studies (72).

In Kris's study, 3 of 26 HER2 exon 20 mutated patients treated with dacomitinib had partial responses, and DCR was $12 \%$, while none of four patients with HER2 amplifications responded to dacomitinib, with PFS of 1,1 , 5 , and 5 months and survival of 5, 7, 15, and 22 months, successively (73). Similar conclusion was drawn in another phase II trial, none of the 13 patients with HER2 YVMA mutant responded. Thus, dacomitinib has limited efficacy in HER2 mutation patients, especially patients with amplification and YVMA mutations could not benefit from it, so patients with these genotypes may not choose dacomitinib (74). However, dacomitinib is shown that it could overcome de novo resistance toward trastuzumab as well as acquired resistance to lapatinib, which may provide solution by combining two drugs together.

Neratinib already showed strong antitumor activity against HER2 amplified and HER2 mutated in vitro and in mice (75). In a phase II trial, neratinib achieved no partial response in NSCLC patients with HER2 mutations, but six patients (35\%) showed stable disease. Eight patients (19\%) resulted a partial response in combination with sirolimus, while 14 patients $(32.5 \%)$ resulted stable disease (76). Neratinib has potential as a therapeutic option for treating HER2-positive NSCLC. Notably, no HER2 exon 20 insertion mutant NSCLC show an objective response to neratinib treatment.

\section{New drugs from ongoing clinical studies}

Considering limited drug selection and poor prognosis of lung cancer patients with HER2 mutation, researchers are undergoing cells level, in vitro, and finally in vivo to develop new drugs to fill the gap.

A new agent conjugated by HER2-specific affibody ZHER2: V2 and pemetrexed inhibited growth of HER2positive lung adenocarcinoma in both cell level and xenografts models $(\mathrm{P}<0.05)$, which indicated potential to be a targeted drug for lung cancer (77). Trastuzumab deruxtecan (DS-8201a) also showed activity in HER2positive solid tumors (78). Meanwhile, FuranopyrimidineBased Epidermal Growth Factor Receptor Inhibitor (DBPR112) shows significant antitumor efficacy in xenograft in vivo models and is currently undergoing phase I clinical trial (79). Tarloxotinib is a hypoxia-activated prodrug of a pan-HER2 kinase inhibitor, has shown preclinical efficacy in EGFR exon 20 and HER2 mutant NSCLC patients. In another study (NCT03805841), 23 patients were treated with tarloxotinib, showing antitumor activity with an ORR of $60 \%$ for all evaluable 20 patients (80).

\section{Discussion}

HER2 mutations are rare in non-small cell lung cancer, only 36 patients were found by NGS, in other words a rate of $3.2 \%$ in a previous retrospective study (81). Also, a proportion of patients have combined mutations, and ratios varied in different studies. Among these 36 patients, six patients $(16.7 \%)$ had a co-existent of EGFR mutation and 
two (5.6\%) had ALK rearrangement.

Studies have explored the superiority among chemotherapy, immunotherapy and targeted therapy. Firstline chemotherapy achieved final PFS of 6 months (95\% CI: 3.97-8.03 months) while first-line immunotherapy was 6 months (95\% CI: 4.17-7.83 months). Also, no statistical difference was found between HER2 20 exon insertion mutation group and HER2 amplification group in terms of PFS or OS. Mazières' study including 101 patients from 38 centers resulted with a median overall survival of 24 months. Overall response rate and median PFS of conventional chemotherapy were $43.5 \%, 6$ months in firstline treatment $(\mathrm{n}=93)$, compared with $10 \%, 4.3$ months in second-line $(\mathrm{n}=52)(46)$. Another study included 38 stage IV HER2-positive lung cancer patients, with an overall survival of 2.3 years (95\% CI: $1.2-2.6$ years). Median survival time of chemotherapy was 4.3 months, including 6.2 months for pemetrexed plus platinum or bevacizumab, 4 months for paclitaxel plus platinum or bevacizumab, 2.6 months for gemcitabine and 3.5 months for vinorelbine. Median duration of HER2-TKIs was 2.2 months (82).

In conclusion, median duration of chemotherapy tends to be longer than HER2-targeted drugs in HER2positive lung cancer. Among HER2-targeted therapy, monoclonal antibodies show a higher response rate than TKIs. Unfortunately, benefit of antibodies or their conjugates is limited to patients with HER2 overexpression or amplification, while no satisfactory results are observed for patients with mutations. For these patients, TKIs and chemotherapy are viable options.

The above conclusions are mostly for patients with adenocarcinoma, while association between HER2 overexpression or amplification and prognosis in patients with squamous cell carcinoma is not found to be statistically significant. Therefore, a unified diagnostic process should be adopted to assess HER2 status in subsequent studies accessing prognosis and therapeutic effect of HER2targeted drugs (83).

Chemotherapy, as a widely used lung cancer therapy in clinic, is often used in comparison or combination with TKIs. Mazières' study (84) included 22 NSCLC patients, who diagnosed with a HER2 in-frame insertion in exon 20 and received targeted and chemotherapy, including trastuzumab, lapatinib, afatinib. Best clinical results are achieved among trastuzumab combined with chemotherapy $(\mathrm{n}=15$; DCR: $96 \%)$ and afatinib monotherapy $(\mathrm{n}=4$; DCR: $100 \%)$. In this study, lapatinib or masitinib are also found ineffective. In song's study, PFS of 7 HER2-positive patients treated with first-line targeted therapy was 2.0 months, and patients treated with first-line chemotherapy was 4.6 months. The DCR of 4 patients treated with afatinib was $75 \%$, and PFS was 4 months (20). For patients with a known HER2 exon 20 insertion mutation, OS tended to be superior in first-line TKI group to that receiving chemotherapy (10.8 vs. 9.8 months, $\mathrm{P}=0.40$ ). However, patients receiving first-line chemotherapy may have a median PFS of 5.9 months, numerically longer than that of HER2-TKI (4.6 months, $\mathrm{P}=0.63$ ) (85).

Compared with chemotherapy, although it is hard to conclude that HER2-targeted drugs have superiority, TKIs still have advantages of less significant side effects and no need for hospitalization. Among TKIs, studies have shown that first-generation EGFR-TKIs are less effective in patients with HER2 mutations. For patients with HER2 mutations, second-generation EGFR-TKIs like afatinib and dacomitinib are better choices than first-generation TKIs. Most of these TKIs have lower response rates than HER2 antibody, except positinib. Investigator conjectured that the high activity of positinib results from its small size and flexible structure, which enables it to bind to the kinase domain. Therefore, positinib may be a better drug for patients who cannot benefit from HER2 antibody, while its side effect must be taken in consideration.

At the same time, chemotherapy remains basis of systemic therapy. Chemotherapy leads to better clinical profits and longer survival to patients with advanced lung cancer with HER2 mutation, especially the most common YVMA subtype. For patients with advanced metastasis, chemotherapy is more helpful to prolong survival time. Xu's study also found that chemotherapies had better efficacy than afatinib and other TKIs for Chinese patients with advanced NSCLC and HER2 mutations (first-line PFS: 5.5 vs. 3.7 months, $\mathrm{P}=0.001$ and second-line PFS: 4.2 vs. 2.0 months, $\mathrm{P}=0.031$ ), especially for most common subtype of YVMA insertion (first-line PFS: 6.0 vs. 2.6 months, $\mathrm{P}=0.008$ and second-line PFS: 4.2 vs. 2.6 months, $\mathrm{P}<0.001)(86)$. Especially for patients with brain metastases, majority of patients receiving HER2-targeted drugs had short or no benefit. However, Lapatinib combined with whole-brain radiation therapy (WBRT) may be a feasible approach in patients with brain metastases in Christo's study (71).

Patients tend to receive first-line chemotherapy than targeted drugs, meanwhile first-line therapy is expected to produce longest treatment time, because patients' performance status usually decreases with development of cancer, so clinical data may be biased toward chemotherapy (82). Also, effect of concurrent radiotherapy 
is often neglected in studies.

Studies are now undergoing in combination with chemotherapy and targeted drug therapy for lung cancer patients with HER2 mutations. Results showed that the combination of afatinib and paclitaxel is more effective than afatinib alone. At the same time, combination of trastuzumab, afatinib and chemotherapy may be beneficial, so patients with HER2-positive NSCLC can also attempt afatinib in combination with chemotherapy if conditions permit.

At present, prognostic significance of HER2 dysregulation remains unclear. It is clinically required to develop individualized diagnosis and treatment programs for different patients. Studies have found that coexistence of EGFR and HER2 leads to a high dependence of tumor and metastatic spread, and studies targeting HRE2 may increase clinical options, prolong patient survival and improve prognosis. Combination of anti-EGFR and anti-HER2 drugs may be a potential strategy to overcome acquired resistance to TKIs $(87,88)$.

\section{Acknowledgments}

Funding: This work was supported by the National Nature Science Foundation of China (81772500); Natural Science Foundation of Jiangsu Province (BK20180139); Department of Science and Technology of Jiangsu Province (BE2019718, BE2019719).

\section{Footnote}

Reporting Checklist: The authors have completed the Narrative Review reporting checklist. Available at http:// dx.doi.org/10.21037/jtd-20-3265

Conflicts of Interest: All authors have completed the ICMJE uniform disclosure form (available at http://dx.doi. org/10.21037/jtd-20-3265). The authors have no conflicts of interest to declare.

Ethical Statement: The authors are accountable for all aspects of the work in ensuring that questions related to the accuracy or integrity of any part of the work are appropriately investigated and resolved.

Open Access Statement: This is an Open Access article distributed in accordance with the Creative Commons Attribution-NonCommercial-NoDerivs 4.0 International
License (CC BY-NC-ND 4.0), which permits the noncommercial replication and distribution of the article with the strict proviso that no changes or edits are made and the original work is properly cited (including links to both the formal publication through the relevant DOI and the license). See: https://creativecommons.org/licenses/by-nc-nd/4.0/.

\section{References}

1. Chi $\mathrm{F}, \mathrm{Wu} \mathrm{R}$, Jin $\mathrm{X}$, et al. HER2 induces cell proliferation and invasion of non-small-cell lung cancer by upregulating COX-2 expression via MEK/ERK signaling pathway. Onco Targets Ther 2016;9:2709-16.

2. Ko YS, Kim NY, Pyo JS. Concordance analysis between HER2 immunohistochemistry and in situ hybridization in non-small cell lung cancer. Int J Biol Markers 2018;33:49-54.

3. Yoshizawa A, Sumiyoshi S, Sonobe M, et al. HER2 status in lung adenocarcinoma: a comparison of immunohistochemistry, fluorescence in situ hybridization (FISH), dual-ISH, and gene mutations. Lung Cancer 2014;85:373-8.

4. Wei XW, Gao X, Zhang XC, et al. Mutational landscape and characteristics of ERBB2 in non-small cell lung cancer. Thorac Cancer 2020;11:1512-21.

5. Liu Z, Wu L, Cao J, et al. Clinical characterization of ERBB2 exon 20 insertions and heterogeneity of outcomes responding to afatinib in Chinese lung cancer patients. Onco Targets Ther 2018;11:7323-31.

6. Yamamoto H, Yatabe Y, Toyooka S. Inherited lung cancer syndromes targeting never smokers. Transl Lung Cancer Res 2018;7:498-504.

7. Krawczyk P, Nicoœ M, Powrózek T, et al. Sensitive methods for the detection of an insertion in exon 20 of the HER2 gene in the metastasis of non-small cell lung cancer to the central nervous system. Oncol Lett 2013;6:1063-7.

8. Chu QS. Targeting non-small cell lung cancer: driver mutation beyond epidermal growth factor mutation and anaplastic lymphoma kinase fusion. Ther Adv Med Oncol 2020;12:1758835919895756.

9. Park CK, Hur JY, Choi CM, et al. Efficacy of Afatinib in a Previously-Treated Patient with Non-Small Cell Lung Cancer Harboring HER2 Mutation: Case Report. J Korean Med Sci 2018;33:e7.

10. Berghoff AS, Magerle M, Ilhan-Mutlu A, et al. Frequent overexpression of ErbB--receptor family members in brain metastases of non-small cell lung cancer patients. Apmis 2013;121:1144-52. 
11. Kim EK, Kim KA, Lee CY, et al. The frequency and clinical impact of HER2 alterations in lung adenocarcinoma. PLoS One 2017;12:e0171280.

12. Díaz-Serrano A, Gella P, Jiménez E, et al. Targeting EGFR in Lung Cancer: Current Standards and Developments. Drugs 2018;78:893-911.

13. Yu HA, Arcila ME, Rekhtman N, et al. Analysis of tumor specimens at the time of acquired resistance to EGFR-TKI therapy in 155 patients with EGFR-mutant lung cancers. Clin Cancer Res 2013;19:2240-7.

14. Gu FF, Zhang Y, Liu YY, et al. Lung adenocarcinoma harboring concomitant SPTBN1-ALK fusion, c-Met overexpression, and HER-2 amplification with inherent resistance to crizotinib, chemotherapy, and radiotherapy. $\mathrm{J}$ Hematol Oncol 2016;9:66.

15. Murtuza A, Bulbul A, Shen JP, et al. Novel ThirdGeneration EGFR Tyrosine Kinase Inhibitors and Strategies to Overcome Therapeutic Resistance in Lung Cancer. Cancer Res 2019;79:689-98.

16. Ramalingam SS, Yang JC, Lee CK, et al. Osimertinib As First-Line Treatment of EGFR Mutation-Positive Advanced Non-Small-Cell Lung Cancer. J Clin Oncol 2018;36:841-9.

17. Li BT, Ross DS, Aisner DL, et al. HER2 Amplification and HER2 Mutation Are Distinct Molecular Targets in Lung Cancers. J Thorac Oncol 2016;11:414-9.

18. Khanal N, Ganti AK. Emerging targeted therapies in non-small cell lung cancer. Expert Rev Anticancer Ther 2016;16:177-87.

19. Liu L, Shao X, Gao W, et al. The role of human epidermal growth factor receptor 2 as a prognostic factor in lung cancer: a meta-analysis of published data. J Thorac Oncol 2010;5:1922-32.

20. Song Z, Yu X, Shi Z, et al. HER2 mutations in Chinese patients with non-small cell lung cancer. Oncotarget 2016;7:78152-8

21. Shen H, Du G, Liu Z, et al. Assessment and prognostic analysis of EGFR mutations or/and HER2 overexpression in Uygur's Non-small Cell Lung Cancer. Int J Clin Exp Med 2015;8:22300-9.

22. Landi L, Cappuzzo F. HER2 and lung cancer. Expert Rev Anticancer Ther 2013;13:1219-28.

23. Peters S, Stahel R, Bubendorf L, et al. Trastuzumab Emtansine (T-DM1) in Patients with Previously Treated HER2-Overexpressing Metastatic Non-Small Cell Lung Cancer: Efficacy, Safety, and Biomarkers. Clin Cancer Res 2019;25:64-72.

24. Gkolfinopoulos S, Mountzios G. Beyond EGFR and ALK: targeting rare mutations in advanced non-small cell lung cancer. Ann Transl Med 2018;6:142.

25. Fang W, Zhao S, Liang Y, et al. Mutation Variants and CoMutations as Genomic Modifiers of Response to Afatinib in HER2-Mutant Lung Adenocarcinoma. Oncologist 2020;25:e545-54.

26. Yuan B, Zhao J, Zhou C, et al. Co-Occurring Alterations of ERBB2 Exon 20 Insertion in Non-Small Cell Lung Cancer (NSCLC) and the Potential Indicator of Response to Afatinib. Front Oncol 2020;10:729.

27. Zhao S, Fang W, Pan H, et al. Conformational Landscapes of HER2 Exon 20 Insertions Explain Their Sensitivity to Kinase Inhibitors in Lung Adenocarcinoma. J Thorac Oncol 2020;15:962-72.

28. Zhang X, Lv J, Wu Y, et al. HER2 Exon 20 Insertion Mutations in Lung Adenocarcinoma: Case Series and Response to Pyrotinib. Front Oncol 2020;10:1162.

29. Fan Y, Qiu J, Yu R, et al. Clinical and molecular characteristics of Chinese non-small cell lung cancer patients with ERBB2 transmembrane domain mutations. Mol Oncol 2020;14:1731-9.

30. Chuang JC, Stehr H, Liang Y, et al. ERBB2-Mutated Metastatic Non-Small Cell Lung Cancer: Response and Resistance to Targeted Therapies. J Thorac Oncol 2017;12:833-42.

31. Da Silva J, Jouida A, Ancel J, et al. FHIT(low) / pHER2(high) signature in non-small cell lung cancer is predictive of anti-HER2 molecule efficacy. J Pathol 2020;251:187-99.

32. Lara PN Jr, Laptalo L, Longmate J, et al. Trastuzumab plus docetaxel in HER2/neu-positive non-small-cell lung cancer: a California Cancer Consortium screening and phase II trial. Clin Lung Cancer 2004;5:231-6.

33. Zinner RG, Glisson BS, Fossella FV, et al. Trastuzumab in combination with cisplatin and gemcitabine in patients with Her2-overexpressing, untreated, advanced non-small cell lung cancer: report of a phase II trial and findings regarding optimal identification of patients with Her2overexpressing disease. Lung Cancer 2004;44:99-110.

34. Gatzemeier U, Groth G, Butts C, et al. Randomized phase II trial of gemcitabine-cisplatin with or without trastuzumab in HER2-positive non-small-cell lung cancer. Ann Oncol 2004;15:19-27.

35. Hotta K, Aoe K, Kozuki T, et al. A Phase II Study of Trastuzumab Emtansine in HER2-Positive Non-Small Cell Lung Cancer. J Thorac Oncol 2018;13:273-9.

36. Hainsworth JD, Meric-Bernstam F, Swanton C, et al. Targeted Therapy for Advanced Solid Tumors on the Basis 
of Molecular Profiles: Results From MyPathway, an OpenLabel, Phase IIa Multiple Basket Study. J Clin Oncol 2018;36:536-42.

37. Cappuzzo F, Gregorc V, Rossi E, et al. Gefitinib in pretreated non-small-cell lung cancer (NSCLC): analysis of efficacy and correlation with HER2 and epidermal growth factor receptor expression in locally advanced or metastatic NSCLC. J Clin Oncol 2003;21:2658-63.

38. Cappuzzo F, Varella-Garcia M, Shigematsu H, et al. Increased HER2 gene copy number is associated with response to gefitinib therapy in epidermal growth factor receptor-positive non-small-cell lung cancer patients. J Clin Oncol 2005;23:5007-18.

39. Hirsch FR, Varella-Garcia M, Cappuzzo F, et al. Combination of EGFR gene copy number and protein expression predicts outcome for advanced non-small-cell lung cancer patients treated with gefitinib. Ann Oncol 2007;18:752-60.

40. Lai WV, Lebas L, Barnes TA, et al. Afatinib in patients with metastatic or recurrent HER2-mutant lung cancers: a retrospective international multicentre study. Eur J Cancer 2019;109:28-35.

41. Fan Y, Chen J, Zhou C, et al. Afatinib in patients with advanced non-small cell lung cancer harboring HER2 mutations, previously treated with chemotherapy: A phase II trial. Lung Cancer 2020;147:209-13.

42. De Grève J, Moran T, Graas MP, et al. Phase II study of afatinib, an irreversible ErbB family blocker, in demographically and genotypically defined lung adenocarcinoma. Lung Cancer 2015;88:63-9.

43. Wang Y, Jiang T, Qin Z, et al. HER2 exon 20 insertions in non-small-cell lung cancer are sensitive to the irreversible pan-HER receptor tyrosine kinase inhibitor pyrotinib. Ann Oncol 2019;30:447-55.

44. Zhou C, Li X, Wang Q, et al. Pyrotinib in HER2-Mutant Advanced Lung Adenocarcinoma After Platinum-Based Chemotherapy: A Multicenter, Open-Label, Single-Arm, Phase II Study. J Clin Oncol 2020;38:2753-61.

45. Robichaux JP, Elamin YY, Vijayan RSK, et al. Pan-Cancer Landscape and Analysis of ERBB2 Mutations Identifies Poziotinib as a Clinically Active Inhibitor and Enhancer of T-DM1 Activity. Cancer Cell 2019;36:444-57.e7.

46. Mazières J, Barlesi F, Filleron T, et al. Lung cancer patients with HER2 mutations treated with chemotherapy and HER2-targeted drugs: results from the European EUHER2 cohort. Ann Oncol 2016;27:281-6.

47. de Langen AJ, Jebbink M, Hashemi SMS, et al. Trastuzumab and paclitaxel in patients with EGFR mutated NSCLC that express HER2 after progression on EGFR TKI treatment. Br J Cancer 2018;119:558-64.

48. Li BT, Shen R, Buonocore D, et al. Ado-Trastuzumab Emtansine for Patients With HER2-Mutant Lung Cancers: Results From a Phase II Basket Trial. J Clin Oncol 2018;36:2532-7.

49. Tsurutani J, Iwata H, Krop I, et al. Targeting HER2 with Trastuzumab Deruxtecan: A Dose-Expansion, Phase I Study in Multiple Advanced Solid Tumors. Cancer Discov 2020;10:688-701.

50. Horvath L, Pircher A. ASCO 2020 non-small lung cancer (NSCLC) personal highlights. Memo 2021. [Epub ahead of print]. doi: 10.1007/s12254-020-00673-2.

51. Ettinger DS, Wood DE, Aisner DL, et al. NCCN Guidelines Insights: Non-Small Cell Lung Cancer, Version 2.2021. J Natl Compr Canc Netw 2021;19:254-66.

52. Ivanova E, Kuraguchi M, Xu M, et al. Use of Ex Vivo Patient-Derived Tumor Organotypic Spheroids to Identify Combination Therapies for HER2 Mutant Non-Small Cell Lung Cancer. Clin Cancer Res 2020;26:2393-403.

53. Scheuer W, Friess T, Burtscher H, et al. Strongly enhanced antitumor activity of trastuzumab and pertuzumab combination treatment on HER2-positive human xenograft tumor models. Cancer Res 2009;69:9330-6.

54. Varella-Garcia M, Mitsudomi T, Yatabe Y, et al. EGFR and HER2 genomic gain in recurrent non-small cell lung cancer after surgery: impact on outcome to treatment with gefitinib and association with EGFR and KRAS mutations in a Japanese cohort. J Thorac Oncol 2009;4:318-25.

55. Xu JM, Han Y, Duan HQ, et al. EGFR mutations and HER2/3 protein expression and clinical outcome in Chinese advanced non-small cell lung cancer patients treated with gefitinib. J Cancer Res Clin Oncol 2009;135:771-82.

56. Soh J, Toyooka S, Ichihara S, et al. Impact of HER2 and EGFR gene status on gefitinib-treated patients with nonsmall-cell lung cancer. Int J Cancer 2007;121:1162-7.

57. Shi Y, Wang M. Afatinib as first-line treatment for advanced lung adenocarcinoma patients harboring HER2 mutation: A case report and review of the literature. Thorac Cancer 2018;9:1788-94.

58. Costa DB, Jorge SE, Moran JP, et al. Pulse Afatinib for ERBB2 Exon 20 Insertion-Mutated Lung Adenocarcinomas. J Thorac Oncol 2016;11:918-23.

59. Peters S, Curioni-Fontecedro A, Nechushtan H, et al. Activity of Afatinib in Heavily Pretreated Patients With ERBB2 Mutation-Positive Advanced NSCLC: Findings 
From a Global Named Patient Use Program. J Thorac Oncol 2018;13:1897-905.

60. Liao BC, Lin CC, Yang JC. Novel EGFR Inhibitors in Non-small Cell Lung Cancer: Current Status of Afatinib. Curr Oncol Rep 2017;19:4.

61. Landi L, Tiseo M, Chiari R, et al. Activity of the EGFRHER2 dual inhibitor afatinib in EGFR-mutant lung cancer patients with acquired resistance to reversible EGFR tyrosine kinase inhibitors. Clin Lung Cancer 2014;15:411-7.e4.

62. Lin L, Ge H, Yan Z, et al. Response to Afatinib in a Patient with Non-Small Cell Lung Cancer Harboring HER2 R896G Mutation: A Case Report. Onco Targets Ther 2019;12:10897-902.

63. Li BT, Lee A, O'Toole S, et al. HER2 insertion YVMA mutant lung cancer: Long natural history and response to afatinib. Lung Cancer 2015;90:617-9.

64. Al-Obeidi E, Li T, Kelly K. Durable Responses to Afatinib as First-line Therapy for HER2-mutated Metastatic Nonsmall-cell Lung Cancer. Clin Lung Cancer 2020;21:e15-20.

65. Moody TW, Lee L, Ramos-Alvarez I, et al. Neurotensin receptors regulate transactivation of the EGFR and HER2 in a reactive oxygen species-dependent manner. Eur J Pharmacol 2019;865:172735.

66. Wu Y, Ni J, Chang X, et al. Successful treatment of pyrotinib for bone marrow metastasis induced pancytopenia in a patient with non-small-cell lung cancer and ERBB2 mutation. Thorac Cancer 2020;11:2051-5.

67. Koga T, Kobayashi Y, Tomizawa K, et al. Activity of a novel HER2 inhibitor, poziotinib, for HER2 exon 20 mutations in lung cancer and mechanism of acquired resistance: An in vitro study. Lung Cancer 2018;126:72-9.

68. Subramanian J, Katta A, Masood A, et al. Emergence of ERBB2 Mutation as a Biomarker and an Actionable Target in Solid Cancers. Oncologist 2019;24:e1303-14.

69. Jasra S, Opyrchal M, Norton L, et al. A Rare Case of S310F Somatic ERBB2 Mutation in a HER2Nonamplified Breast Cancer. Clin Breast Cancer 2017;17:e37-41.

70. Ramlau R, Thomas M, Novello S, et al. Phase I Study of Lapatinib and Pemetrexed in the Second-Line Treatment of Advanced or Metastatic Non-Small-Cell Lung Cancer With Assessment of Circulating Cell Free Thymidylate Synthase RNA as a Potential Biomarker. Clin Lung Cancer 2015;16:348-57.

71. Christodoulou C, Kalogera-Fountzila A, Karavasilis $\mathrm{V}$, et al. Lapatinib with whole brain radiotherapy in patients with brain metastases from breast and non- small cell lung cancer: a phase II study of the Hellenic Cooperative Oncology Group (HeCOG). J Neurooncol 2017;134:443-51.

72. Ross HJ, Blumenschein GR, Jr., Aisner J, et al. Randomized phase II multicenter trial of two schedules of lapatinib as first- or second-line monotherapy in patients with advanced or metastatic non-small cell lung cancer. Clin Cancer Res 2010;16:1938-49.

73. Kris MG, Camidge DR, Giaccone G, et al. Targeting HER2 aberrations as actionable drivers in lung cancers: phase II trial of the pan-HER tyrosine kinase inhibitor dacomitinib in patients with HER2-mutant or amplified tumors. Ann Oncol 2015;26:1421-7.

74. Takeda M, Sakai K, Hayashi H, et al. Clinical characteristics of non-small cell lung cancer harboring mutations in exon 20 of EGFR or HER2. Oncotarget 2018;9:21132-40.

75. Ogoshi Y, Shien K, Yoshioka T, et al. Anti-tumor effect of neratinib against lung cancer cells harboring HER2 oncogene alterations. Oncol Lett 2019;17:2729-36.

76. Rothschild SI. Targeted Therapies in Non-Small Cell Lung Cancer-Beyond EGFR and ALK. Cancers (Basel) 2015;7:930-49.

77. Jiao $\mathrm{H}$, Zhao X, Liu J, et al. In vivo imaging characterization and anticancer efficacy of a novel HER2 affibody and pemetrexed conjugate in lung cancer model. Nucl Med Biol 2019;68-69:31-9.

78. Tamura K, Tsurutani J, Takahashi S, et al. Trastuzumab deruxtecan (DS-8201a) in patients with advanced HER2positive breast cancer previously treated with trastuzumab emtansine: a dose-expansion, phase 1 study. Lancet Oncol 2019;20:816-26.

79. Lin SY, Chang Hsu Y, Peng YH, et al. Discovery of a Furanopyrimidine-Based Epidermal Growth Factor Receptor Inhibitor (DBPR112) as a Clinical Candidate for the Treatment of Non-Small Cell Lung Cancer. J Med Chem 2019;62:10108-23.

80. Liu SV, Villaruz LC, Lee VHF, et al. LBA61 First analysis of RAIN-701: Study of tarloxotinib in patients with nonsmall cell lung cancer (NSCLC) EGFR Exon 20 insertion, HER2-activating mutations \& other solid tumours with NRG1/ERBB gene fusions. Ann Oncol 2020;31:S1189.

81. Lee K, Jung HA, Sun JM, et al. Clinical Characteristics and Outcomes of Non-small Cell Lung Cancer Patients with HER2 Alterations in Korea. Cancer Res Treat 2020;52:292-300.

82. Eng J, Hsu M, Chaft JE, et al. Outcomes of chemotherapies and HER2 directed therapies in advanced 
HER2-mutant lung cancers. Lung Cancer 2016;99:53-6.

83. Heydt C, Michels S, Thress KS, et al. Novel approaches against epidermal growth factor receptor tyrosine kinase inhibitor resistance. Oncotarget 2018;9:15418-34.

84. Mazières J, Peters S, Lepage B, et al. Lung cancer that harbors an HER2 mutation: epidemiologic characteristics and therapeutic perspectives. J Clin Oncol 2013;31:1997-2003.

85. Zhou J, Ding N, Xu X, et al. Clinical outcomes of patients with HER2-mutant advanced lung cancer: chemotherapies versus HER2-directed therapies. Ther Adv Med Oncol 2020;12:1758835920936090.

86. Xu F, Yang G, Xu H, et al. Treatment outcome and

Cite this article as: Wu R, Yuan B, Li C, Wang Z, Song Y, Liu $\mathrm{H}$. A narrative review of advances in treatment and survival prognosis of HER2-positive malignant lung cancers. J Thorac Dis 2021;13(6):3708-3720. doi: 10.21037/jtd-20-3265 clinical characteristics of HER2 mutated advanced nonsmall cell lung cancer patients in China. Thorac Cancer 2020;11:679-85.

87. Hsu CC, Liao BC, Liao WY, et al. Exon 16-Skipping HER2 as a Novel Mechanism of Osimertinib Resistance in EGFR L858R/T790M-Positive Non-Small Cell Lung Cancer. J Thorac Oncol 2020;15:50-61.

88. Suzawa K, Toyooka S, Sakaguchi M, et al. Antitumor effect of afatinib, as a human epidermal growth factor receptor 2-targeted therapy, in lung cancers harboring HER2 oncogene alterations. Cancer Sci 2016;107:45-52. 